\title{
Next-Generation Sequencing in Clinical Practice: Is It a Cost-Saving Alternative to a Single-Gene Testing Approach?
}

\author{
Giancarlo Pruneri $^{1,2} \cdot$ Filippo De Braud $^{3,4}$. Anna Sapino ${ }^{5,6} \cdot$ Massimo Aglietta $^{7,8}$. Andrea Vecchione ${ }^{9}$. \\ Raffaele Giusti ${ }^{10}$. Caterina Marchiò ${ }^{5,6}$. Stefania Scarpino ${ }^{9}$. Anna Baggi ${ }^{11}$ (1) . Giuseppe Bonetti ${ }^{11}$. \\ Jean Marie Franzini ${ }^{11} \cdot$ Marco Volpe $^{11} \cdot$ Claudio Jommi $^{12}$
}

Accepted: 12 December 2020 / Published online: 4 March 2021

(c) The Author(s) 2021

\begin{abstract}
Objectives This study aimed to compare the costs of a next-generation sequencing-based (NGS-based) panel testing strategy to those of a single-gene testing-based (SGT-based) strategy, considering different scenarios of clinical practice evolution. Methods Three Italian hospitals were analysed, and four different testing pathways (paths 1, 2, 3, and 4) were identified: two for advanced non-small-cell lung cancer (aNSCLC) patients and two for unresectable metastatic colon-rectal cancer (mCRC) patients. For each path, we explored four scenarios considering the current clinical practice and its expected evolution. The 16 testing cases ( 4 scenarios $\times 4$ paths) were then compared in terms of differential costs between the NGS-based and SGT-based approaches considering personnel, consumables, equipment, and overhead costs. Break-even and sensitivity analyses were performed. Data gathering, aimed at identifying the hospital setup, was performed through a semi-structured questionnaire administered to the professionals involved in testing activities.

Results The NGS-based strategy was found to be a cost-saving alternative to the SGT-based strategy in 15 of the 16 testing cases. The break-even threshold, the minimum number of patients required to make the NGS-based approach less costly than the SGT-based approach, varied across the testing cases depending on molecular alterations tested, techniques adopted, and specific costs. The analysis found the NGS-based approach to be less costly than the SGT-based approach in nine of the 16 testing cases at any volume of tests performed; in six cases, the NGS-based approach was found to be less costly above a threshold (and in one case, it was found to be always more expensive). Savings obtained using an NGS-based approach ranged from $€ 30$ to $€ 1249$ per patient; in the unique testing case where NGS was more costly, the additional cost per patient was $€ 25$. Conclusions An NGS-based approach may be less costly than an SGT-based approach; also, generated savings increase with the number of patients and different molecular alterations tested.
\end{abstract}

\section{Introduction and Research Question}

Next-generation sequencing (NGS) is a technology that allows the simultaneous sequencing of selected regions of the genome [targeted sequencing (TS)] up to the whole exome [whole exome sequencing (WES)] or whole genome [whole genome sequencing (WGS)] [1,2]. NGS is currently used to identify mutations for diagnostic and therapeutic purposes in clinical practice (CP) or in the context of specifically designed clinical trials [3].

Growing interest in NGS has raised the question of its value for money (cost-effectiveness) and sustainability

Claudio Jommi

claudio.jommi@unibocconi.it

Extended author information available on the last page of the article

\section{Key Points for Decision Makers}

Testing strategies for advanced non-small-cell lung cancer and unresectable metastatic colon-rectal cancer patients can vary among Italian hospitals in terms of molecular alterations tested and testing techniques used.

Decreasing the adoption of single-gene testing (SGT) techniques in favour of next-generation sequencing (NGS) use can lead to cost reduction. Testing a minimum number of patients and molecular alterations might be necessary to generate savings.

The number of different molecular alterations to be tested is expected to grow in the near future along with the potential savings generated by the use of NGS. 
(budget impact) compared to the traditional single-gene testing (SGT) approach.

In Italy, the SGT-based approach is usually carried out in laboratories where the NGS technology is not available, and consists of detecting those biomarkers that are druggable with treatments approved and reimbursed by the Italian Drug Agency (AIFA). For example, advanced non-small-cell lung cancer (aNSCLC) patients can be treated with specific anti-epidermal growth factor receptor (anti-EGFR), antianaplastic lymphoma kinase (anti-ALK), or anti-receptor tyrosine kinase (anti-ROS) drugs; these biomarkers can be tested with single tests based on real-time polymerase chain reaction (RT-PCR), single-gene sequencing, in situ hybridization techniques, and immunohistochemistry. Alternatively, the NGS-based approach aims at simultaneously evaluating all the actionable biomarkers regardless of AIFA approval and reimbursement.

Three recent papers investigated the challenges posed by economic evaluations applied to NGS [4] and systematically reviewed the most recent evidence on the economic impact of the NGS-based versus SGT-based approach [5, 6]. For cost estimates, these reviews found huge variations in both methods (e.g. gross costing vs bottom-up approach) and their findings. Some studies estimated the comprehensive diagnostic pathway costs (including patient consultations and admissions), while others focused on genetic sequencing. The reviews raised criticisms not only for the heterogeneity of methods used, but also because many cost analyses did not present data transparently and did not fully declare the cost items included [5], thus limiting data comparability and transferability. Review authors advocated for the production of guidelines on the costing of diagnostic pathways [6]. The main limitations found in the studies included in the reviews are that they (1) rarely relied on microcosting, which is particularly required for testing in heterogeneous patient populations; (2) were mostly retrospective analyses, whose completeness depends on data availability; (3) represented a broad clinical spectrum of genetic disorders; (4) were often carried out on a small sample size; and (5) adopted a simplistic approach, assuming that NGS may substitute all current procedures adopted as SGT.

The present study aimed to compare the costs of the NGS-based approach with different TS panels to those of the SGT-based approach, overcoming most of the limitations raised for other studies and adopting a dynamic approach, i.e. comparing different testing scenarios, where NGS with different TS panels is gradually integrated into the SGTbased approach. Furthermore, we estimated the threshold, in terms of the number of patients, where the NGS-based approach becomes less costly than the SGT-based approach.

The analysis has been carried out in Italy, where NGS testing is currently not reimbursed at the national level, since it is not included in the Italian basket of healthcare goods and services centrally defined by the national Ministry of Health. However, the regions that are responsible for the delivery of healthcare and the relevant budget may provide health services beyond this level at their own expenses [7]. Since inpatient and outpatient services, including diagnostic testing, are paid on a fee-for-episode basis, reimbursement of NGS at the regional level would require that the Regional Government set the relevant fee. According to a report published in 2017, this has happened only in the largest Italian region (Lombardy) so far (€2072) [8]. The same report illustrates the availability of NGS sequencers in four regions, accounting for $35 \%$ of the Italian population: at least one NGS sequencer in $40 \%$ of hospitals (our elaboration on [8, 9]), with an estimate of one sequencer per 2.4 per million inhabitants. There is not, to our best knowledge, a nationwide analysis of the use of NGS technologies in Italy.

The current analysis focused on non-small-cell lung cancer (NSCLC) and unresectable metastatic colon-rectal cancer (mCRC). NSCLC is the first and third leading cause of tumour-related deaths in Italy for men (26\% of deaths) and women ( $11 \%$ of deaths), respectively. The diagnosis frequently occurs in advanced stages (aNSCLC), where the current therapies have limited efficacy, contributing to poor outcomes (15.8\% 5-year survival rate) [10]. Unresectable mCRC is the second most frequent cancer in Italy both in terms of incidence and deaths, with a 5-year survival rate of $66 \%$ (colon cancer) and 62\% (rectal cancer) [10]. Both diseases can be treated with targeted therapies, but the future role played by NGS-based techniques has been differently envisaged, with an expected increasing importance for NSCLC $[11,12]$ and higher uncertainty for mCRC.

\section{Methods}

The comparison between the SGT-based and NGS-based approaches in molecular alteration testing focused on aNSCLC and mCRC patients (first- and second-line treatments), with $45 \%$ of patients treated with second-line therapy for aNSCLC [13] and $49.5 \%$ of patients treated with second-line therapy for mCRC [14]. The cost analysis was conducted considering the hospital perspective.

The molecular alterations considered were as follows:

- aNSCLC: epidermal growth factor receptor (EGFR), kirsten rat sarcoma viral oncogene (KRAS), mesenchymal-epithelial transition factor (MET) exon 14 skipping, and B-Raf and V-Raf murine sarcoma viral oncogene homolog $\mathrm{B}(B R A F)$ mutations; anaplastic lymphoma kinase $(A L K)$, ROS proto-oncogene 1 receptor tyrosine kinase (ROS-1), and rearranged during transfection (RET) fusions; mesenchymal-epithelial transition factor (MET) amplification; human epidermal growth factor 
receptor 2 (HER2) status (copy number alteration or mutation, protein expression); programmed death-ligand 1 (PD-L1) status; microsatellite instability (MSI); and tumour mutational burden (TMB);

- mCRC: KRAS, neuroblastoma rat sarcoma viral oncogene (NRAS), and BRAF mutations; PD-L1 status; O6-methylguanine DNA methyltransferase $(M G M T)$ promoter methylation; MutL homolog 1 (MLH1), MutS homolog 2 (MSH2), postmeiotic segregation increased 2 (PMS2), and MutS homolog 6 (MSH6) protein expression; HER2 and $E G F R$ status (copy number alteration, protein expression); MSI; RET and NTRK1/NTRK2/NTRK3 pan neurotrophic tropomyosin receptor kinase (PANTRK) fusions; fibroblast growth factor receptor (FGFR2) gene status; and TMB.

Three Italian hospitals were selected through convenience sampling [15] based on hospital willingness to participate and expertise in the diseases considered:

- Scientific Institute for Research, Hospitalisation and Health Care (IRCCS) Istituto Nazionale dei Tumori (INT) (Milan), which was the pilot centre supporting the research protocol design (aNSCLC and mCRC tests accounted for $60 \%$ of the laboratory activity)

- Teaching Hospital Trust Sant'Andrea (Rome) for aNSCLC (whose tests accounted for $75 \%$ of the laboratory activity)

- IRCCS Istituto Candiolo (Turin) for mCRC (whose tests accounted for $10 \%$ of the laboratory activity).

Only one hospital was considered for both aNSCLC and mCRC; hence, four testing pathways (paths 1, 2, 3, and 4) were investigated, two for each target disease (Fig. 1).

The number of aNSCLC patients tested per year were 364 and 317 for paths 1 and 2, respectively; mCRC patients tested per year were 260 and 225 for paths 3 and 4, respectively (2016-2018 average).

Input data were collected through interviews based on a semi-structured questionnaire and administered to a CRC/ NSCLC oncologist, pathologist, biologist/molecular biologist, or laboratory technician. Aggregated data were collected without tracking patient-specific information. The pilot hospital (INT) was more intensively involved, with six interviews conducted for each path. Information was organized in a data collection template, validated with followup interviews, and cross-validated with the interviewees through a direct check of the template. For the other hospitals, a single interview was conducted with all the involved healthcare professionals, and a similar approach to the INT review was used. Volume and cost data were collected by responders; time spent performing testing was derived from a perceptual response based on laboratory staff experience.
For each path, four scenarios were considered:

- Clinical Practice (CP) scenario (i.e. current testing pathway).

- "Minimum set" scenario, in which molecular tests were carried out if strongly recommended by the Italian oncological guidelines $[16,17]$. This scenario is usually more conservative than the $\mathrm{CP}$ scenario.

- "Future CP without TMB" scenario, in which gene testing was expanded to mutations that are expected to be routinely included in the future, excluding TMB, i.e. a measurement of the mutation load carried by the tumour cells that represents a putative predictive biomarker for immune therapy [18].

- "Future $C P$ " scenario, in which the CP scenario was extended to mutations that are expected to be included in future as well as TMB analysis.

The four scenarios were analysed for each of the four paths. For the resulting 16 testing cases, two alternative testing approaches were compared:

- SGT-based approach, where molecular alterations were tested with single-gene techniques [e.g. fluorescence in situ hybridization (FISH), polymerase chain reaction (PCR), Sanger sequencing, immunohistochemistry] and NGS was used only if standard techniques were unsuitable (e.g. for TMB testing).

- NGS-based approach, where molecular alterations were tested, as much as possible, through NGS panel testing; otherwise, SGT techniques were adopted.

A comparison of the NGS-based and SGT-based approaches was conducted as a variation in the transition from an SGT-based approach to an NGS-based approach (hereafter referred to as $\Delta_{\mathrm{SGT} \rightarrow \mathrm{NGS}}$ ).

The cost items considered were personnel, consumables, equipment (purchasing and maintenance), and overheads; cost data refer to the year 2018-2019.

For each technique and molecular alteration, the time spent by each healthcare professional involved in the testing activities was investigated. Given the proportion of fixed and variable time defined for each activity, the overall personnel time absorbed varied with the average machine saturation (number of samples per run). Saturation depended on the volumes of tests performed, maximum number of samples per run, laboratory opening hours, and required time for result delivery (14 calendar days) [19-21]. Considering the machine time required to perform the test and personnel reporting activities, 8 working days was assumed as the deadline to setup the machine and begin the run.

The personnel time absorbed was monetized using the hourly gross salary (€61670/year, €61601/year, and €73047/ 
Fig. 1 Research design. Two testing pathways for each of the diseases considered (paths 1 and 2 for aNSCLC and paths 3 and 4 for $\mathrm{mCRC}$ ) were investigated in three Italian hospitals. Four scenarios ("minimum set", "clinical practice" (CP), "future CP no TMB", and "future CP") were defined for each testing pathway, and cost analyses were conducted comparing the SGT-based and NGS-based approaches for each of the 16 testing cases identified. aNSCLC advanced non-small-cell lung cancer, $C P$ clinical practice, $m C R C$ metastatic colon-rectal cancer, $N G S$ next-generation sequencing, $S G T$ single-gene testing, $T M B$ tumour mutational burden, $v s$ versus

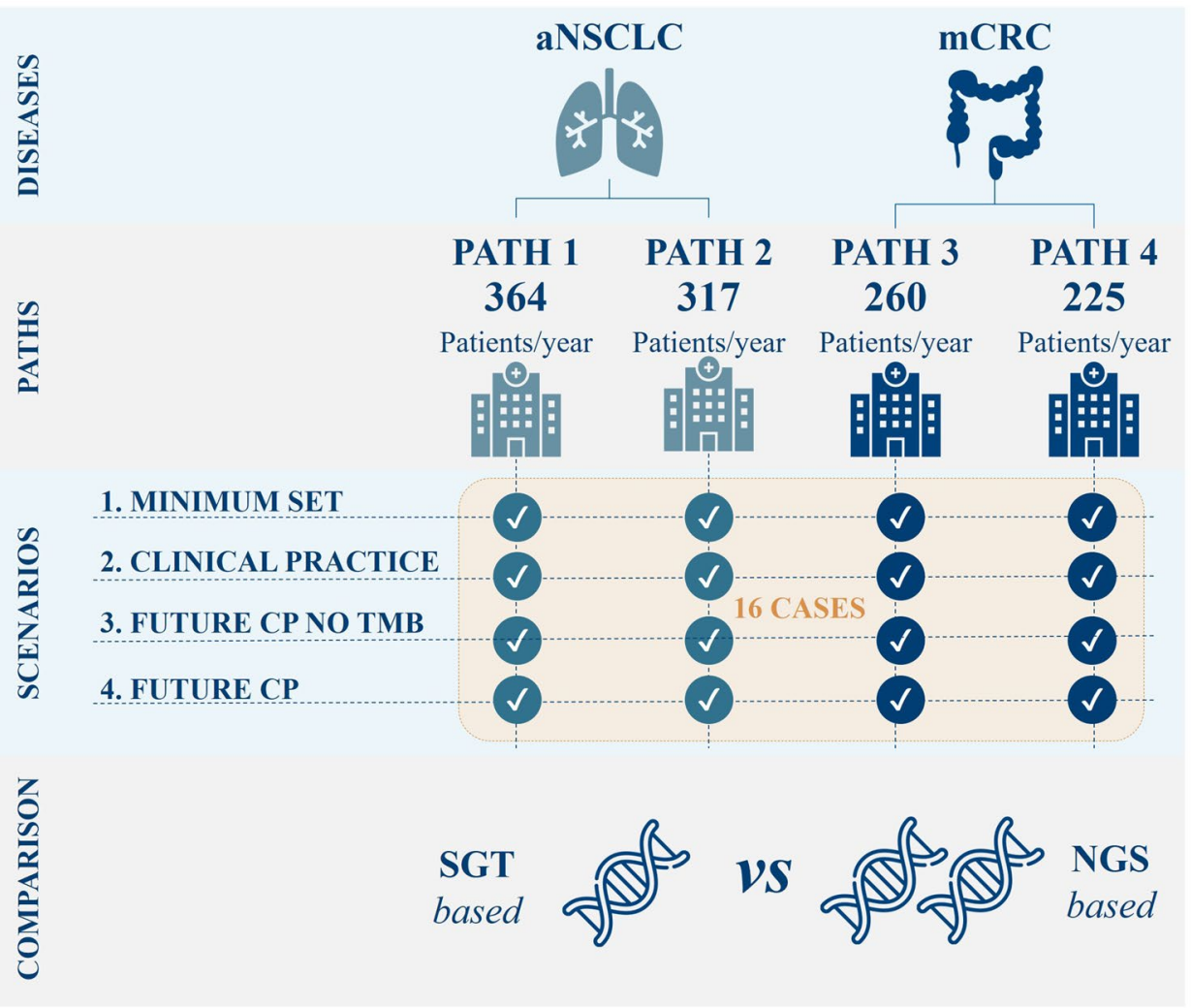

year for laboratory technicians, biologists, and pathologists, respectively) [22].

Consumables costs were provided for each combination of techniques and molecular alterations tested. Unit costs [including institutional discounts and value-added tax (VAT)] were obtained from purchase orders, reflecting the actual costs sustained by the hospitals. In particular, NGS consumables costs refer to the Ion Torrent ${ }^{\mathrm{TM}}$ Ion S5 System, provided by one of the involved hospitals and validated by the other two.

Equipment purchasing costs were provided by the accounting team of one of the involved hospitals. As for the consumables, NGS equipment costs refer to the Ion Torrent ${ }^{\mathrm{TM}}$ Ion S5 System. The other two hospitals did not provide their own data, but confirmed that those provided could be used as a reference in the analysis. An alternative approach was identified in the adoption of publicly available tenders for Italian hospitals (years 2017-2018), ${ }^{1}$ but the data were too variable to be considered. Investment depreciation was set to 5 years [8], while the annual maintenance cost was assumed to be $10 \%$ [23] of the machine purchasing cost. Since aNSCLC/mCRC testing partially accounted for the total machine activity, the proportion of time dedicated to performing aNSCLC/mCRC tests for each technique was applied, and acquisition costs were re-proportionated accordingly (Annex Table 1, see the electronic supplementary material). We assigned to each scenario only those equipment and maintenance costs that refer to the specific techniques used.

The hospitals involved did not provide the overhead costs, and even if they had been provided, the different allocation approaches in use (e.g. level of data aggregation, cost items included, allocation drivers) would have made it difficult to obtain a sound comparison of the specific amount allocated to each technique. Furthermore, there is no reference in the Italian literature that can provide guidelines on overheads allocation in our specific case. Therefore, we decided to adopt the approach used by Schwarze et al., where total costs were increased by an additional $20 \%$ to account for overheads [24].

For each path, an evaluation with varying volumes of tested patients was performed to identify the minimum number of patients beyond which the NGS-based approach became less costly (break-even analysis).

Single-way deterministic sensitivity analysis (DSA) was performed for the CP scenario to identify the key model drivers. Each parameter was varied by $\pm 20 \%$. Savings generated by NGS adoption increased in more advanced scenarios, thus the choice of performing the analysis in the $\mathrm{CP}$ scenario can be considered a conservative approach.

${ }_{1}$ Supplementary material on request. 


\section{Results}

The results section reports (1) an overview of the input data (further information is reported in the annex, see the electronic supplementary material), (2) the mean cost per patient, (3) the personnel time and cost absorbed for testing activities, (4) the capacity increase, (5) the break-even analysis, and (6) the sensitivity analysis.

The results are based on the $\Delta_{\mathrm{SGT} \rightarrow \mathrm{NGS}}$ comparison, and a discussion is presented in the sections below. A complete illustration of the results achieved for each testing case is provided in Table 1.

\subsection{Input Data}

The analysis considered the following for each path:

- Molecular alterations tested and testing frequency upon the first and second treatment lines in each scenario (Annex Table 2, see the electronic supplementary material)

- Testing techniques for each molecular alteration, including NGS (Annex Table 3, see the electronic supplementary material)

- Validation and retesting frequency for each molecular alteration in case of failure or an inconclusive result and the type of technique adopted (Annex Table 4, see the electronic supplementary material)

- Time required to perform testing and reporting activities for each healthcare professional involved (Annex Table 5, see the electronic supplementary material)

- Equipment costs including purchase, depreciation and maintenance costs (Annex Table 1, see the electronic supplementary material)

- Consumables costs for each technique and molecular alteration tested (Annex Table 6, see the electronic supplementary material)

- Overhead costs calculated as percentage (20\%) of the resulting total cost.

Participating hospitals differed in terms of molecular alterations currently tested, expected evolution of the testing approach, and techniques adopted. These differences led to a different average number of tests per patient (Table 2), ranging from one (path 4), meaning that all the alterations are tested with the NGS panel test, to nine (path 3) for the NGSbased technique, and from 1.3 (path 4) to 12.5 (path 3) for the SGT-based technique. Moving from the "minimum set" to the "future CP" scenario, the number of tests per patient increased, although to a lesser extent, using the NGS-based approach, which allows simultaneous testing. The remarkable difference between path 3 and path 4 in terms of average number of tests per patient is mainly due to the considerably wider set of molecular alterations tested in path 3 compared to path 4 (Table 2).

\subsection{Mean Cost Per Patient}

The mean cost per patient, including personnel, consumables, equipment, and overhead costs, is reported in Fig. 2.

The $\Delta_{\mathrm{SGT} \rightarrow \mathrm{NGS}}$ savings per patient increased in scenarios with a larger set of molecular alterations tested. Savings in the "minimum set" and "future CP" scenarios ranged from $€ 288$ to $€ 879$, from $-€ 25$ (the only case of a cost increment) to $€ 522$, from $€ 63$ to $€ 633$, and from $€ 30$ to $€ 1249$ for paths $1,2,3$, and 4, respectively.

The NGS-based approach reduced personnel costs in all testing cases. The consumables $\Delta_{\mathrm{SGT} \rightarrow \mathrm{NGS}}$ costs were reduced in all of the testing cases except for path 2 in the "minimum set" and CP scenarios. In path 2, less expensive SGT techniques were preferred to more expensive techniques adopted by other hospitals, making the $\Delta_{\mathrm{SGT} \rightarrow \mathrm{NGS}}$ savings more difficult to achieve. Consumables were the most relevant cost item, and the savings achieved compensated for the potential increase in equipment costs. Equipment costs increased from the "minimum set" scenario to the "future CP" scenario since more molecular alterations need to be tested and more testing techniques need to be used. Overhead costs are proportional to the total cost of personnel, consumables, and equipment. The NGS capacity to test multiple molecular alterations caused the NGS-based approach to be less costly than the SGT-based approach in scenarios with more comprehensive testing, generating equipment cost savings in half of the testing cases considered.

The overall cost comparison is discussed in the following sections and is summarized in Table 1.

\subsection{Personnel Time}

$\Delta_{\text {SGT } \rightarrow \text { NGS }}$ personnel time savings increased progressively in scenarios based on more comprehensive testing.

The $\Delta_{\mathrm{SGT} \rightarrow \mathrm{NGS}}$ overall personnel time savings were $628 \mathrm{~h}$, $271 \mathrm{~h}, 103 \mathrm{~h}$, and $333 \mathrm{~h}$ for paths 1, 2, 3, and 4, respectively, in the "minimum set" scenario and $1369 \mathrm{~h}, 691 \mathrm{~h}, 470 \mathrm{~h}$, and 919 h, respectively, in the "future CP" scenario (Fig. 3).

The overall time savings for each path are reported in Fig. 3. Personnel cost savings, as reported in Table 1, were proportional to time savings, ranging from $€ 3618$ (path 3, "minimum set") to $€ 48,216$ (path 1, "future CP").

Personnel time saved was converted into the number of additional patients who could be potentially tested by the hospital (Table 3) using an NGS-based approach, providing an estimation of the capacity increase. This indicator was calculated by dividing the overall personnel time savings 


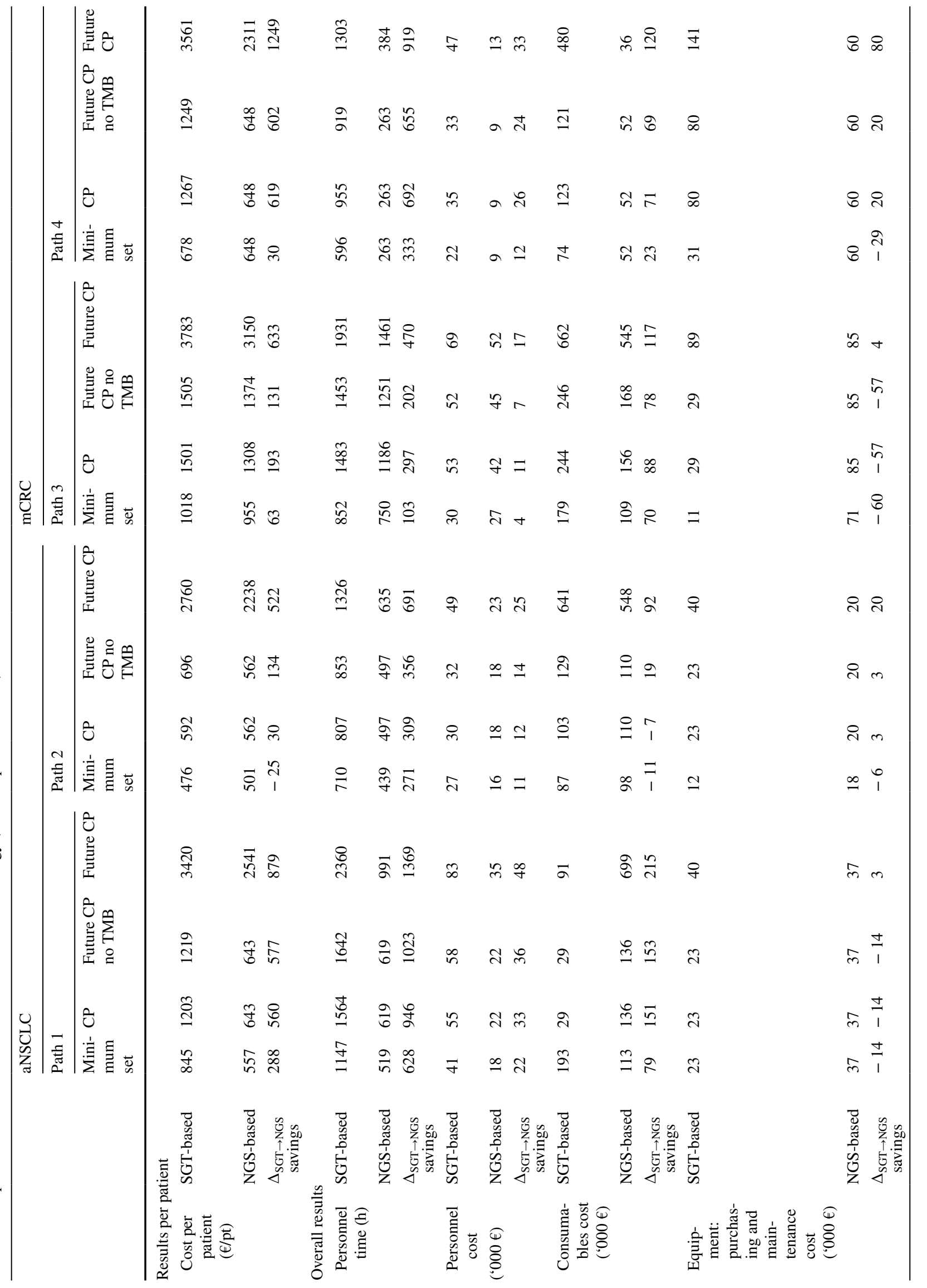




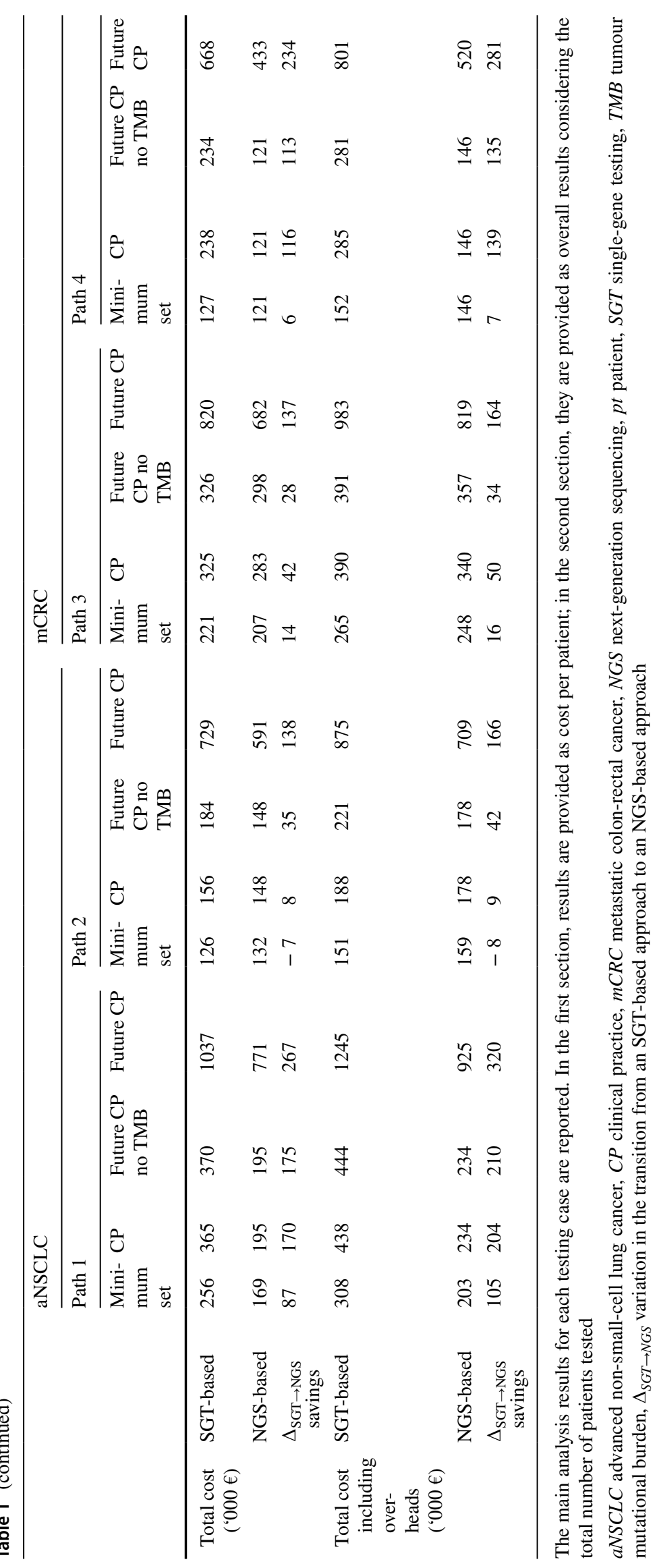


Table 2 Average number of tests per patient. Each path differs in terms of molecular alterations tested and testing frequency in the respective scenarios and in terms of testing techniques used, thus, resulting in a specific number of tests per patient

\begin{tabular}{|c|c|c|c|c|c|c|c|c|}
\hline & \multicolumn{4}{|c|}{ Path 1: aNSCLC } & \multicolumn{4}{|c|}{ Path 2: aNSCLC } \\
\hline & Minimum set & $\mathrm{CP}$ & $\begin{array}{l}\text { Future CP no } \\
\text { TMB }\end{array}$ & Future $\mathrm{CP}$ & Minimum set & $\mathrm{CP}$ & $\begin{array}{l}\text { Future CP no } \\
\text { TMB }\end{array}$ & Future $\mathrm{CP}$ \\
\hline SGT-based & 5.2 & 6.7 & 7.2 & 8.2 & 4.6 & 5.2 & 5.6 & 6.7 \\
\hline NGS-based & 2.1 & 2.1 & 2.1 & 2.1 & 2.1 & 2.6 & 2.6 & 2.6 \\
\hline \multirow[t]{3}{*}{$\Delta_{\mathrm{SGT} \rightarrow \mathrm{NGS}}$} & 3.1 & 4.6 & 5.1 & 6.1 & 2.5 & 2.6 & 3.0 & 4.1 \\
\hline & \multicolumn{4}{|l|}{ Path 3: mCRC } & \multicolumn{4}{|l|}{ Path 4: mCRC } \\
\hline & Minimum set & $\mathrm{CP}$ & $\begin{array}{l}\text { Future CP no } \\
\text { TMB }\end{array}$ & Future $\mathrm{CP}$ & Minimum set & $\mathrm{CP}$ & $\begin{array}{l}\text { Future CP no } \\
\text { TMB }\end{array}$ & Future $\mathrm{CP}$ \\
\hline SGT-based & 7.1 & 11.6 & 11.5 & 12.5 & 1.3 & 2.6 & 2.5 & 3.5 \\
\hline NGS-based & 5.1 & 8.4 & 9.0 & 9.0 & 1.0 & 1.0 & 1.0 & 1.0 \\
\hline$\Delta_{\mathrm{SGT} \rightarrow \mathrm{NGS}}$ & 2.0 & 3.2 & 2.5 & 3.5 & 0.3 & 1.6 & 1.5 & 2.5 \\
\hline
\end{tabular}

aNSCLC advanced non-small-cell lung cancer, $C P$ clinical practice, $m C R C$ metastatic colon-rectal cancer, $N G S$ next-generation sequencing, $S G T$ single-gene testing, $T M B$ tumour mutational burden, $\Delta_{S G T \rightarrow N G S}$ variation in the transition from an SGT-based approach to an NGS-based approach

Path 1 - aNSCLC

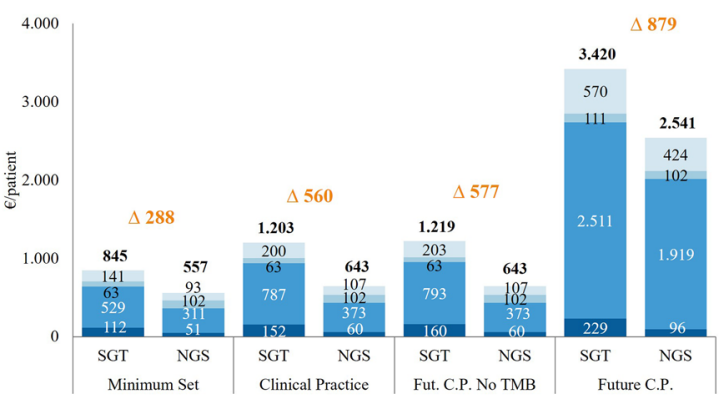

Path 3 - mCRC

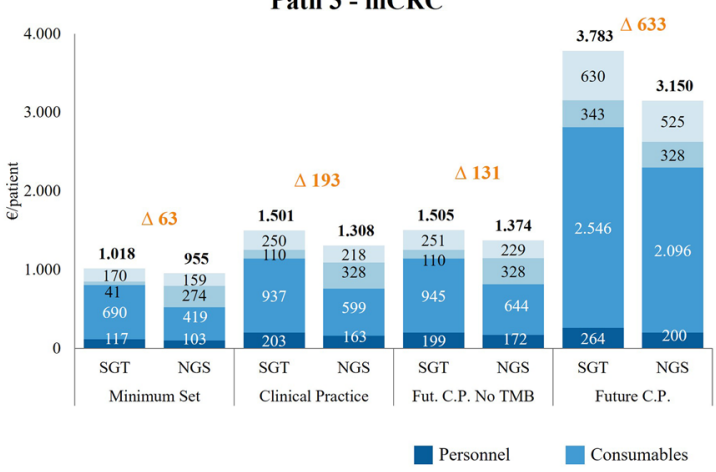

Fig. 2 Mean cost per patient. The mean cost per patient comparing the SGT-based and NGS-based approaches is shown for each testing case considering personnel, consumables, equipment (purchasing and maintenance), and overhead costs to perform all the required tests.
Path 2 - aNSCLC

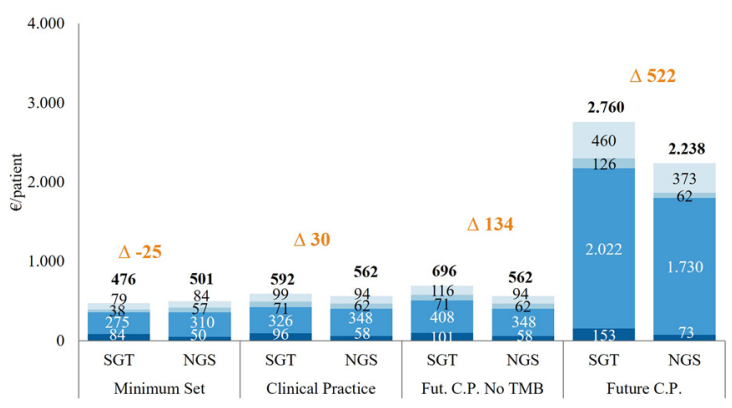

Path 4 - mCRC

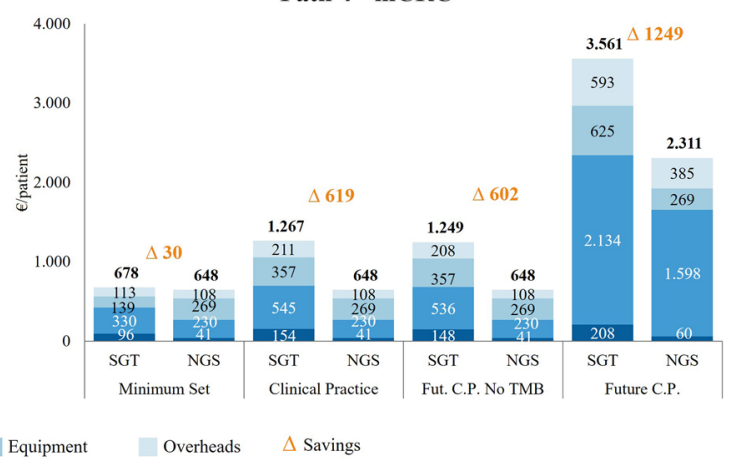

aNSCLC advanced non-small-cell lung cancer, $C P$ clinical practice, $m C R C$ metastatic colon-rectal cancer, $N G S$ next-generation sequencing, $S G T$ single-gene testing, $T M B$ tumour mutational burden 
Fig. $3 \Delta_{\mathrm{SGT} \rightarrow \mathrm{NGS}}$ overall personnel time savings. The overall personnel time savings generated by the NGS-based approach are shown for each testing case. aNSCLC advanced non-small-cell lung cancer, $C P$ clinical practice, $m C R C$ metastatic colon-rectal cancer, $N G S$ next-generation sequencing, $S G T$ single-gene testing, TMB tumour mutational burden
Path 1 - aNSCLC

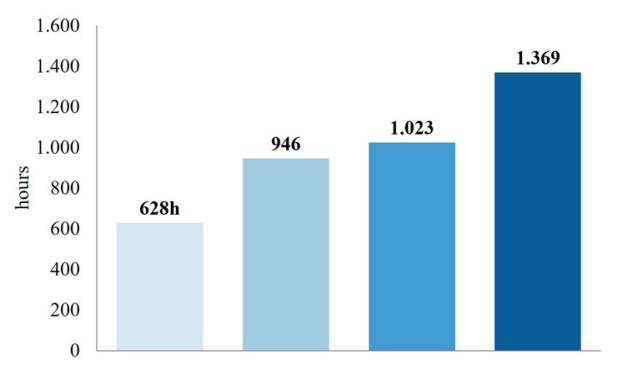

Path 3 - mCRC

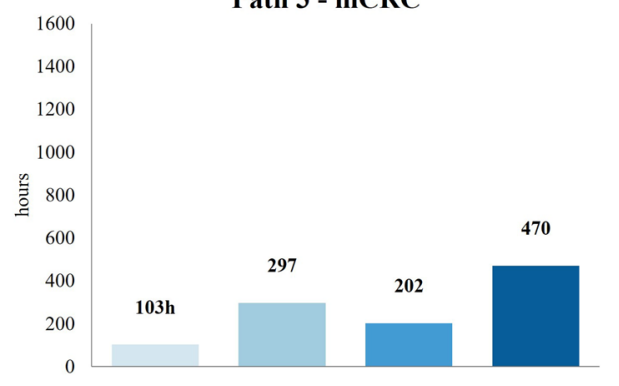

Minimum set Clinical Practice
Path 2 - aNSCLC

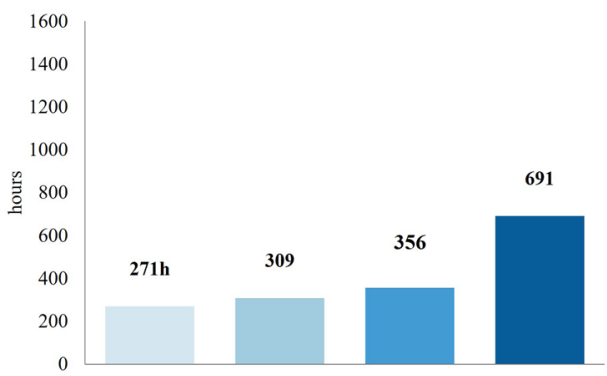

Path 4 - mCRC

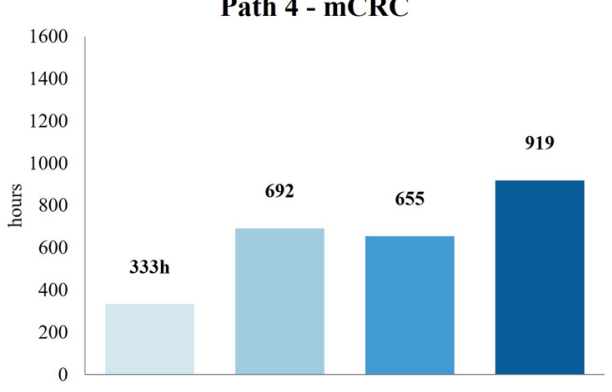

Future CP no TMB

Future CP by the average personnel time spent performing all the tests required for a patient in the NGS-based approach.

Additional testable patients generated by the NGS-based approach ranged from 506, 142, 36, and 206 for paths 1, 2, 3 , and 4, respectively, in the "minimum set" scenario, and a maximum of $923,294,56$, and 397 , in the "future CP" scenario.

\subsection{Break-Even Analysis}

The break-even volume represents the number of tested patients where the SGT-based cost per patient equals the NGS-based cost; above this threshold, an NGS-based approach generates savings.

Break-even charts for path 3 are presented in Fig. 4, while Table 4 illustrates the volumes and break-even thresholds for all paths. The larger the number of tested molecular alterations, the more the testing process can benefit from the NGS capacity to test a wider range of molecular alterations in a single run; the SGT-based approach would need to involve different techniques, hence requiring a higher number of tests. Table 4 shows that, moving from the "minimum set" to the "future CP" scenario, the patient break-even volume (if applicable) decreases and the NGS savings are easier to achieve.

\subsection{Deterministic Sensitivity Analysis}

Deterministic sensitivity analyses were performed for each path in the $\mathrm{CP}$ scenario, where all key parameters were varied by $\pm 20 \%$. In all the paths, consumables costs (used for the Sanger technique in paths 1 and 3 and for NGS or mass spectrometry for paths 2 and 4) were the most impactful variable, as shown in Fig. 5. The variation within the determined range of the most impactful parameter (impact on $\Delta_{\text {SGT } \rightarrow \text { NGS }}$ savings is $\pm € 39,183, \pm € 17,667, \pm € 26,760$, and $\pm € 16,200$ for paths $1,2,3$, and 4 , respectively) caused the SGT-based approach to be slightly less expensive (€8215) than the NGS-based approach in path 2 only.

\section{Discussion}

The introduction of NGS has enhanced the potential detection of mutations, with an important impact on the diagnosis and treatment of many diseases. Although diagnostic procedures represent a small proportion of total pathway costs, growing attention has been paid to the impact of NGS on the budget and its cost-effectiveness compared to the standard single-testing approach. Three recent papers on economic studies on NGS have raised concerns on the way cost analyses have been carried out [4-6]. Furthermore, they revealed that the evidence on NGS costs is quite poor for Europe, and no data exist for Italy. 
Table 3 Overall additional patients due to $\Delta_{\text {SGT } \rightarrow \text { NGS }}$ time savings

\begin{tabular}{lllll}
\hline & \multicolumn{4}{l}{ Capacity increase (patients) } \\
\cline { 2 - 5 } & Minimum set & CP & $\begin{array}{l}\text { Future CP } \\
\text { no TMB }\end{array}$ & Future CP \\
\hline Path 1-aNSCLC & 506 & 786 & 852 & 923 \\
Path 2-aNSCLC & 142 & 119 & 164 & 294 \\
Path 3-mCRC & 36 & 43 & 27 & 56 \\
Path 4-mCRC & 206 & 321 & 294 & 397 \\
\hline
\end{tabular}

Additional patients who could be potentially tested in each testing case if $\Delta_{\mathrm{SGT} \rightarrow \mathrm{NGS}}$ personnel time savings were used to increase the testing activity and if the NGS-based approach was adopted

aNSCLC advanced non-small-cell lung cancer, $C P$ clinical practice, $m C R C$ metastatic colon-rectal cancer, $N G S$ next-generation sequencing, $S G T$ single-gene testing, $T M B$ tumour mutational burden, $\Delta_{S G T \rightarrow N G S}$ variation in the transition from an SGT-based approach to an NGS-based approach

The present study aimed to cover the information gap for Italy, investigating the costs of different diagnostic approaches (NGS-based vs SGT-based) in three Italian hospitals according to different scenarios characterized by an increasing number of mutations analysed. The current study was planned before the two systematic reviews were published. Notwithstanding, it was designed to address most of the limitations of previous studies that the two reviews highlighted. Our analysis compared different scenarios (dynamic approach) where NGS was gradually implemented and was not a virtual comparison between a full adoption of NGS and single testing. A microcosting approach was adopted because this approach is suggested if cost analyses are carried out in heterogeneous patients. We also detailed the cost items and methods used for estimation. Another advantage of the current study is that the mean cost per patient was estimated instead of the mean cost per test, to take into account possible double testing.

According to our estimates, the diagnostic mean cost per patient ranges from $€ 501$ to $€ 3150$ for the NGS-based approach and from $€ 476$ to $€ 3783$ for the SGT-based approach. Our results are comparable to those from most other studies relying on microcosting analyses and applied to cancer care. Two studies were conducted in the USA [25] and the Netherlands [26]. The relevant estimates included pre-analytics and analytics labour, equipment and consumables, bioinformatic data analysis, reporting, and overhead costs. Depending on the procedure used, the full assay cost per sample ranged between US\$699 (€655 [27]) and US\$2428 (€2275 [27]) in the USA and between
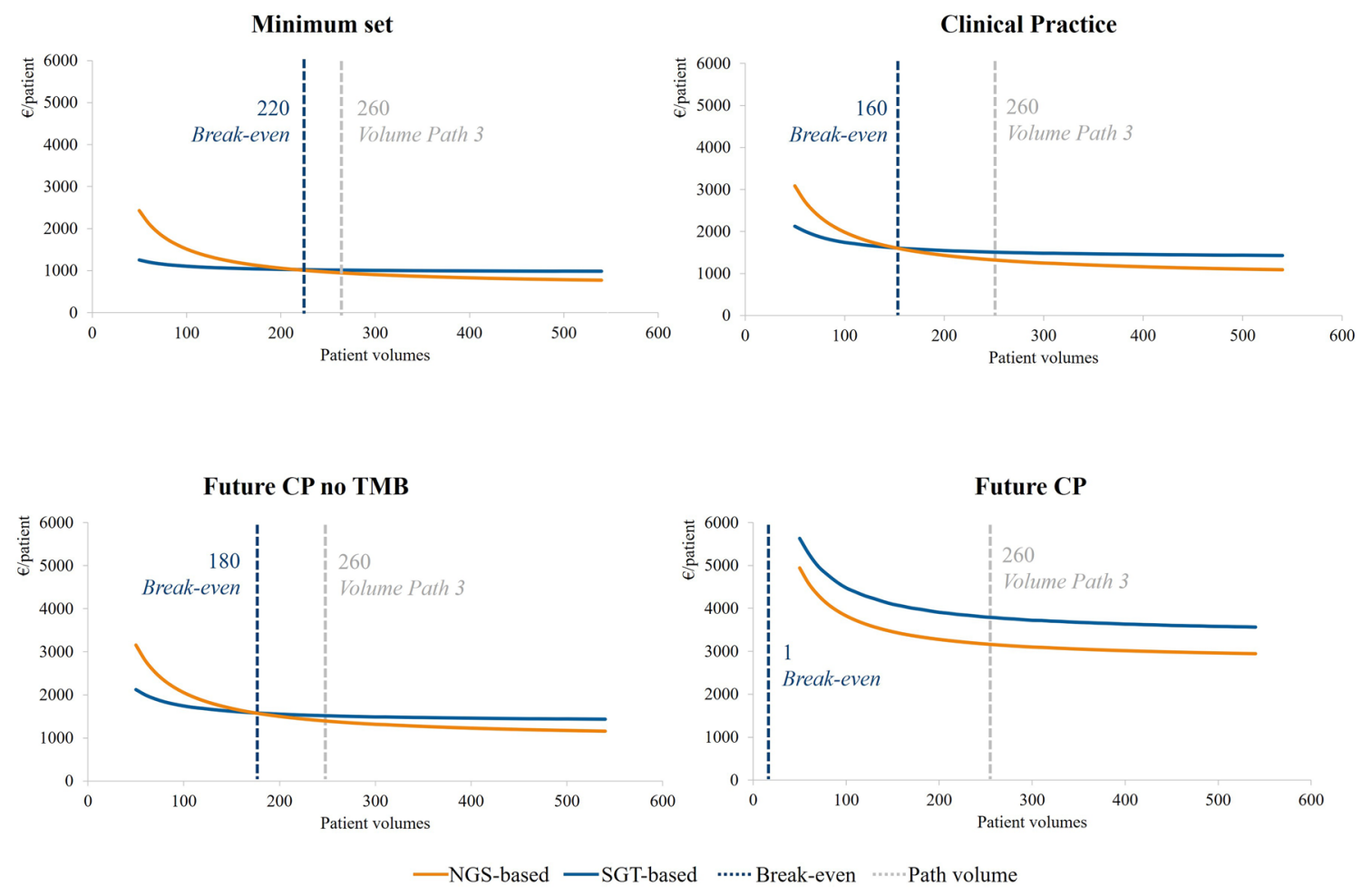

Fig. 4 Break-even analysis (path 3). The mean cost per patient for the SGT-based and NGS-based approaches in path 3 is displayed for each scenario and reported as a function of patient volume. The number of patients tested by the hospital ("volume path 3") and the threshold necessary for the NGS-based approach to generate savings ("breakeven") are shown. $C P$ clinical practice, $N G S$ next-generation sequencing, $S G T$ single-gene testing, $T M B$ tumour mutational burden 
Table 4 Break-even analysis volumes

\begin{tabular}{lllllll}
\hline Disease & Path & $\begin{array}{l}\text { Hospital volume } \\
\text { (patients/year) }\end{array}$ & Break-even volume (patients) & \\
\cline { 5 - 7 } & & & Minimum set & CP & $\begin{array}{l}\text { Future CP no } \\
\text { TMB }\end{array}$ & Future CP \\
\hline aNSCLC & 1 & 364 & 60 & $>0$ & $>0$ & $>0$ \\
& 2 & 317 & - & 70 & $>0$ & $>0$ \\
mCRC & 3 & 260 & 220 & 160 & 180 & $>0$ \\
& 4 & 225 & 180 & $>0$ & $>0$ & $>0$ \\
\hline
\end{tabular}

The table illustrates, for each testing case, the break-even volume; " $>0$ " represents the NGS-based approach being less expensive for any number of patients tested

aNSCLC advanced non-small-cell lung cancer, $C P$ clinical practice, $m C R C$ metastatic colon-rectal cancer, $N G S$ next-generation sequencing, $T M B$ tumour mutational burden
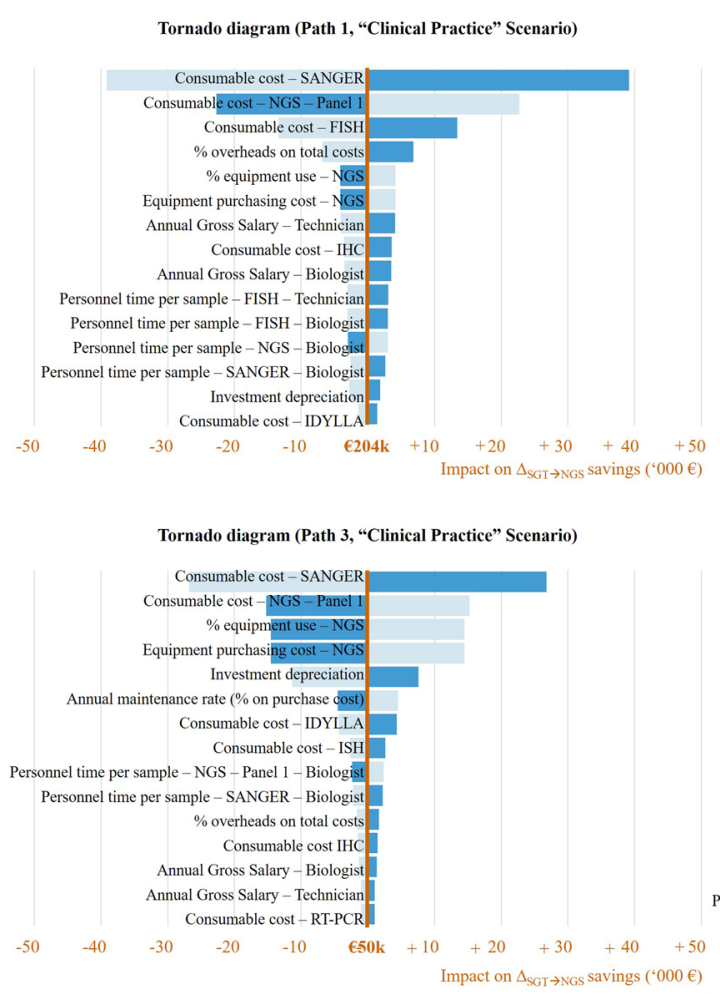

Fig. 5 Deterministic sensitivity analysis (CP scenario). The results of the DSA are shown in the CP scenario and are displayed in a tornado diagram (the 15 most impactful variables are included). Input data were varied $( \pm 20 \%)$, and impacts on the $\Delta_{\text {SGT } \rightarrow \text { NGS }}$ savings were observed. $C P$ clinical practice, $D S A$ deterministic sensitivity analysis, FISH fluorescence in situ hybridization, IHC immunohistochemistry,

$€ 606$ and $€ 2668$ in the Netherlands. The Dutch study also assumed high productivity rates: 24 samples per test, one test per week, and $85 \%$ conclusive results (no additional tests required). Another recent study carried out in a single centre in the UK estimated the cost of genome sequencing for a
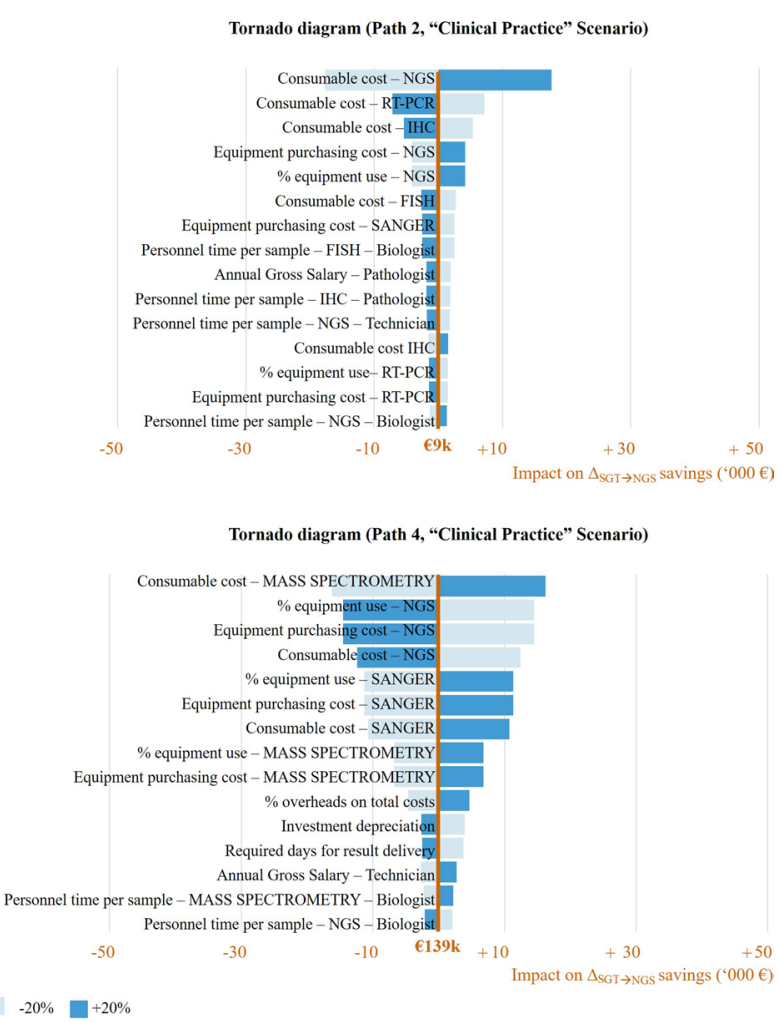

$I S H$ in situ hybridization, $m C R C$ metastatic colon-rectal cancer, $N G S$ next-generation sequencing, $R T-P C R$ real-time polymerase chain reaction, $S G T$ single-gene testing, $T M B$ tumour mutational burden, $\Delta_{S G T \rightarrow N G S}$ variation in the transition from an SGT-based approach to an NGS-based approach

cancer case equal to £6841 (€7754 [28]), £3420 (€3876 [28]) per genome. In all studies, consumables represent the most important cost item [24].

Our analysis reveals that by moving from the SGT-based to the NGS-based approach, hospitals can reduce sequencing 
costs by $€ 30-€ 1249$ per patient depending on the scenario, and only in one testing case is the SGT-based approach less costly. The savings per patient tested are higher in scenarios with a more comprehensive set of molecular alterations tested. Hence, the higher the number of mutations screened, the more the NGS-based approach is less costly than the SGT-based one. This result was not surprising, since the SGT-based approach is less suitable when different alterations should be found. Another important result is that in all testing cases, the personnel time dedicated to sequencing is reduced, thus providing the opportunity to reallocate this saved time in other more labour-intensive activities.

Our research provides another interesting finding, i.e. the minimum number of patients needed to make the NGS-based approach less costly than the SGT-based one. The breakeven point ranged from 0 to 220 depending on the complexity of the analysis, the hospital, and the disease (aNSCLC and $\mathrm{mCRC}$ ). Other studies have shown only that the mean assay cost significantly decreased with higher volumes [26].

These findings have important implications for hospitals and regulators. There is clear evidence that using the NGSbased versus SGT-based approach is advantageous from an economic perspective, provided that a minimum number of patients are screened. This evidence should address the selection of centres accredited for providing NGS-based diagnostic procedures for the whole population.

Most of the other studies have estimated the cost per sample, which is useful for other purposes, i.e. comparing the economic efficiency of different laboratories or determining the fee for service payers should pay laboratories. Having estimated in our study the cost per patient may also help convert a fee-for-service-based funding system into a feefor-episode-based funding system. A fee-for-episode-based funding system may push healthcare providers to better manage the whole sequencing/diagnostic process.

We are aware that the current study has some limitations. The microcosting analysis was applied to a small sample of research-oriented and teaching hospitals, which were selected on the grounds of their willingness to participate in the survey (sample of convenience). Although these hospitals cannot therefore be considered prototypical of Italian hospitals, these centres are likely to represent, in the future, accredited reference laboratories for NGS-based techniques. It should also be noted that two of the three studies relying on the microcosting approach mentioned before were carried out in one single centre [24, 26]. Another limitation of our study is that most of the input data were retrieved from interviews with healthcare professionals due to limited accessibility to other analyses (e.g. time and motion analysis for the time dedicated by each healthcare professional). However, confirmatory, repeated interviews were carried out to cross-check the robustness of the answers. Furthermore, we had to rely on benchmark data for those centres who did not provide all the required information (e.g. equipment purchasing costs and NGS consumables costs for path 2 and path 4) and on international published data for overhead costs, since data for the three centres were not available and there is no available evidence for Italy. Another limitation is the retrospective nature of the study; the most recent systematic review on health economics studies applied to NGS has recommended a prospective analysis [5]. Finally, this is a partial analysis comparing two alternative approaches (NGSbased vs SGT-based) in different scenarios from a cost perspective. This result should be integrated with process (e.g., diagnostic yield) and health (e.g., quality-adjusted life years) outcomes to have a complete set of information for decisionmakers. A cost-effectiveness analysis was beyond our scope and would have required reliable data on the two sequencing strategies in all scenarios considered.

Despite these limitations, our study provides insights into the cost of NGS in Italian laboratories comprehensively testing mutations in aNSCLC and mCRC.

\section{Conclusion}

Although diagnostic procedures do not represent the most relevant cost of cancer patient treatment, they will increasingly play a central role in clinical decision-making. The optimization of diagnostic procedures is important for at least two reasons: (1) mutation-driven treatments have steadily increased in recent years, and (2) tumour-agnostic treatments based on molecular alterations occurring across a variety of different tumour types have been recently approved by the Food and Drug Administration (FDA) [29].

Along this line, our study showed that the NGS-based approach is less costly than the SGT-based approach, provided that a minimum number of patients are tested. This result can be considered for regulatory purposes, including the rules to accredit hospitals for NGS analysis, as well as for access issues (e.g., whether NGS can be covered by thirdparty payers), and finally for economic analysis, since NGS testing may accelerate the use of medicines and investment decisions.

We are perfectly aware that the decision to adopt an NGS approach should consider not only costs but also the effectiveness, organizational impact, availability of suitable treatments, its use in $\mathrm{CP}$ or in clinical trials, and data confidentiality issues, among other factors. Nevertheless, the present analysis aimed to generate evidence on one of the key drivers of decision-making in healthcare.

Supplementary Information The online version contains supplementary material available at https://doi.org/10.1007/s41669-020-00249-0. 
Acknowledgements We would like to thank all the authors for their contribution to the definition of the study design. Special thanks also go to Giovanni Gancitano (Roche Italy), Roberto Scalamogna (Roche Italy), and Adrian Hegenbarth (Business Integration Partners, Life Sciences Division).

\section{Declarations}

Funding The current study was conducted thanks to an unconditional sponsorship from Roche Diagnostics S.p.A.

Conflict of interests The authors have no other conflicts of interest to declare.

Ethics approval Not applicable.

Consent to participate Not applicable.

Consent for publication Not applicable.

Availability of data and material All data inputs used in the analysis are available within the article and the supplementary material.

Code availability Not applicable.

Authors' contributions Conceived and designed the research approach: Giancarlo Pruneri, Filippo De Braud, Anna Sapino, Massimo Aglietta, Andrea Vecchione, Raffaele Giusti, Caterina Marchiò, Stefania Scarpino, Anna Baggi, Giuseppe Bonetti, Jean Marie Franzini, Marco Volpe, and Claudio Jommi. Provided and validated the input data: Giancarlo Pruneri, Filippo De Braud, Anna Sapino, Massimo Aglietta, Andrea Vecchione, Raffaele Giusti, Caterina Marchiò, and Stefania Scarpino. Designed the analytical model and analysed the data: Anna Baggi, Giuseppe Bonetti, Jean Marie Franzini, Marco Volpe, and Claudio Jommi. Interpretation of the analysis: Giancarlo Pruneri, Anna Baggi, Giuseppe Bonetti, Jean Marie Franzini, Marco Volpe, and Claudio Jommi. Wrote the document: Giancarlo Pruneri, Anna Baggi, Giuseppe Bonetti, and Claudio Jommi.

Open Access This article is licensed under a Creative Commons Attribution-NonCommercial 4.0 International License, which permits any non-commercial use, sharing, adaptation, distribution and reproduction in any medium or format, as long as you give appropriate credit to the original author(s) and the source, provide a link to the Creative Commons licence, and indicate if changes were made. The images or other third party material in this article are included in the article's Creative Commons licence, unless indicated otherwise in a credit line to the material. If material is not included in the article's Creative Commons licence and your intended use is not permitted by statutory regulation or exceeds the permitted use, you will need to obtain permission directly from the copyright holder. To view a copy of this licence, visit http://creativecommons.org/licenses/by-nc/4.0/.

\section{References}

1. Vrijenhoek T, Kraaijeveld K, Elferink M, et al. Next-generation sequencing-based genome diagnostics across clinical genetics centers: implementation choices and their effects. Eur J Hum Genet. 2015;23(9):1142-50.

2. Zhao X, Wang A, Walter V, et al. Combined targeted DNA sequencing in non-small cell lung cancer (NSCLC) using UNCseq and NGScopy, and RNA sequencing using UNCqeR for the detection of genetic aberrations in NSCLC. PLOS ONE. 2015;10(6):e0129280.

3. Torshizi AD, Wang K. Next-generation sequencing in drug development: target identification and genetically stratified clinical trials. Drug Discov Today. 2018;23(10):1776-83.

4. Phillips KA, Deverka PA, Marshall DA. Methodological issues in assessing the economic value of next-generation sequencing tests: many challenges and not enough solutions. Value Health. 2018;21(9):1033-42.

5. Fahr P, Buchanan J, Wordsworth S. a review of health economic studies comparing traditional and massively parallel sequencing diagnostic pathways for suspected genetic disorders. Pharmacoeconomics. 2020;38:143-58.

6. Schwarze K, Buchanan J, Taylor JC, Wordsworth S. Are whole-exome and whole-genome sequencing approaches costeffective? A systematic review of the literature. Genet Med. 2018;20(10):1122-30.

7. Ferré F, de Belvis AG, Valerio L, Longhi S, Lazzari A, Fattore G, Ricciardi W, Maresso A. Italy: health system review. Health Syst Transit. 2014;16(4):1-168.

8. Jefferson T, Cerbo M, Chiarolla E, Di Maria E, Favarato M, Gillespie F, Lo Scalzo A, Pinotti G, Turchetti D, Perrini MR, Agenas, HTA report, next generation sequencing (NGS), Rome, 2017.

9. Furnari A, Gugiatti A, Petracca F. La struttura e le attività del SSN. CERGAS, Rapporto OASI 2017, Egea Ed, Milano; 2018.

10. AIOM-AIRTUM, I numeri del cancro in Italia; 2018.

11. Blumenthal GM, Mansfield E, Pazdur R. Next-generation sequencing in oncology in the era of precision medicine. JAMA Oncol. 2016;2(1):13-4.

12. Dienstmann R, Ciner A, Hochster HS. Should next-generation sequencing testing be routinely used in metastatic colorectal cancer? Lancet Oncol. 2018;19(11):1434-5.

13. Stinchcombe M, et al. Consideration for 2 nd line therapy on NSCLC. Oncologist. 2008;13(1):28-36.

14. Datamonitor Healthcare's proprietary colorectal cancer survey, July 2016.

15. Lohr S. Sampling: design and analysis (2nd edition). J Biopharm Stat. 2011;21:175-6.

16. Associazione Italiana di Oncologia Medica (AIOM), "Linee guida neoplasie del polmone," 2018.

17. Associazione Italiana di Oncologia Medica (AIOM), "Linee guida tumori del colon," 2018.

18. Chan TA, Yarchoan M, Jaffee E, et al. Development of tumor mutation burden as an immunotherapy biomarker: utility for the oncology clinic. Ann Oncol. 2019;30(1):44-56.

19. Gruppo di Lavoro di AIOM e SIAPEC-IAP. Raccomandazioni AIOM e SIAPEC-IAP per l'analisi mutazionale del gene EGFR nel carcinoma polmonare. 2014.

20. Gruppo di Lavoro di AIOM e SIAPEC-IAP. Raccomandazioni AIOM e SIAPEC-IAP per la valutazione delle mutazioni di RAS nel carcinoma del colon-retto. 2015.

21. Gruppo di Lavoro di AIOM e SIAPEC-IAP. Raccomandazione per l'analisi dei riarrangiamenti del gene ALK nel carcinoma polmonare non a piccole cellule. 2012.

22. ARAN Agenzia per la Rappresentanza Negoziale delle Pubbliche Amministrazioni.

23. Bektemur G, Muzoglu N, Arici MA, Karaaslan MK. Cost analysis of medical device spare parts. Pak J Med Sci. 2018;34:472-7.

24. Schwarze K, Buchanan J, Jilles M, et al. The complete costs of genome sequencing: a microcosting study in cancer and rare diseases from a single center in the United Kingdom. Genet Med. 2020;22(1):85-94.

25. Sabatini LM, Mathews C, Ptak D, et al. Genomic sequencing procedure microcosting analysis and health economic cost-impact 
analysis: a report of the association for molecular pathology. $\mathrm{J}$ MolDiagn. 2016;18(3):319-28.

26. Van Amerongen RA, Retèl VP, Coupé VMH, et al. Next-generation sequencing in NSCLC and melanoma patients: a cost and budget impact analysis. Ecancermedicalscience. 2016;10:684.

27. Internal Revenue Service. Average $\$$ to $€$ exchange rate for year 2015: 0,937.
28. Gov.UK. Average $£$ to $€$ exchange rate for year 2018: 1,1334.

29. Lemery S, Keegan P, First PR. FDA approval agnostic of cancer site-when a biomarker defines the indication. N Engl J Med. 2017;377(15):1409-12.

\section{Authors and Affiliations}

\section{Giancarlo Pruneri ${ }^{1,2}$. Filippo De Braud ${ }^{3,4} \cdot$ Anna Sapino $^{5,6} \cdot$ Massimo Aglietta $^{7,8} \cdot$ Andrea Vecchione $^{9}$. Raffaele Giusti ${ }^{10}$. Caterina Marchiò ${ }^{5,6}$. Stefania Scarpino ${ }^{9}$. Anna Baggi ${ }^{11}$ (D) . Giuseppe Bonetti ${ }^{11}$. Jean Marie Franzini ${ }^{11} \cdot$ Marco Volpe $^{11} \cdot$ Claudio Jommi ${ }^{12}$ (i)}

1 Department of the Pathology and Laboratory Medicine, Fondazione IRCCS-Istituto Nazionale dei Tumori, Milan, Italy

2 School of Medicine, University of Milan, Milan, Italy

3 Department of Oncology, Fondazione IRCCS-Istituto Nazionale dei Tumori, Milan, Italy

4 School of Medicine, University of Milan, Milan, Italy

5 Pathology Unit, Candiolo Cancer Institute-FPO-IRCCS-Cand iolo, Turin, Italy

6 Department of Medical Sciences, University of Turin, Turin, Italy

7 Medical Oncology, Candiolo Cancer Institute-FPO-IRCCS-C andiolo, Turin, Italy

8 Department of Oncology, University of Torino, Turin, Italy
9 Pathology Unit, Department of Clinical and Molecular Medicine, St. Andrea University Hospital, University of Rome La Sapienza, Rome, Italy

10 Medical Oncology Unit, St. Andrea University Hospital, Rome, Italy

11 Life Sciences Division, Business Integration Partners, Milan, Italy

12 Cergas, Centre for Research on Health and Social Care Management, SDA Bocconi School of Management, Bocconi University, Milan, Italy 